\title{
NUEVAS CITAS DE BULBOSTYLIS (CYPERACEAE) PARA LAS FLORAS DE BOLIVIA Y PARAGUAY
}

\author{
MARÍA GABRIELA LÓPEZ1
}

\begin{abstract}
Summary: López, M. G. 2006. New records of Bulbostylis (Cyperaceae) for the flora of Bolivia and Paraguay. Bonplandia 15(3-4): 149-160. ISSN: 0524-0476.

During the study of the genus Bulbostylis (Cyperaceae, Abildgaardieae) for Austral America, five new records were found, one from Bolivia: $B$. svensoniana Steyerm. and four from Paraguay: B. consanguinea (Kunth) C.B.Clarke, B. loefgrenii (Boeck.) Prata \& López, B. sellowiana (Kunth) Palla and B. papillosa Kük. All the taxa are described and illustrated and considerations about them are given.
\end{abstract}

Key words: Taxonomy, Abildgaardieae, South America.

Resumen: López, M. G. 2006. Nuevas citas de Bulbostylis (Cyperaceae) para las floras de Bolivia y Paraguay. Bonplandia 15(3-4): 149-160. ISSN: 0524-0476.

Durante la revisión del género Bulbostylis (Cyperaceae, Abildgaardieae) para la flora de América austral, se encontraron cinco nuevas citas, una de Bolivia: B. svensoniana Steyerm. y cuatro de Paraguay: B. consanguinea (Kunth) C.B.Clarke, B. loefgrenii (Boeck.) Prata \& López, $B$. sellowiana (Kunth) Palla y $B$. papillosa Kük. Se hacen aclaraciones sobre estos taxones, los cuales se describen e ilustran.

Palabras clave: Taxonomía, Abildgaardieae, Sudamérica.

\section{Introducción}

El género Bulbostylis Kunth (Cyperaceae, Abildgaardieae) cuenta con ca. 150 especies que habitan en regiones tropicales y subtropicales de ambos hemisferios. Algunas de ellas llegan a zonas templadas, siendo su límite de distribución los $40^{\circ}$ de latitud S. Presenta dos centros de diversidad importantes, uno en África y otro en Sudamérica en el centro de Brasil. En Sudamérica viven aproximada- mente 65 especies.

Son plantas heliófilas que habitan en suelos arenosos, húmedos, muy comunes en campos «cerrados» y espartillares, entre rocas. Algunas especies crecen en suelos modificados y se las encuentra como malezas de cultivos.

Como parte de la revisión del género Bulbostylis que se está realizando para la flora de América austral, se encontraron cinco nuevas citas. Una de ellas para Bolivia, Bulbostylis svensoniana Steyerm., y cuatro especies de Paraguay: $B$. consanguinea

${ }^{1}$ Facultad de Ciencias Agrarias, Instituto de Botánica del Nordeste, Casilla de Correo 209, 3400 Corrientes, Argentina. E-mail: mglopez@agr.unne.edu.ar 
(Kunth) C.B. Clarke, B. loefgrenii (Boeck.) Prata \& López, B. sellowiana (Kunth) Palla y B. papillosa Kük.

Bulbostylis svensoniana hasta el momento era conocida para Venezuela, Paraguay (Kral, 1998) y Brasil, en los estados de Mato Grosso do Sul, Goiás, Minas Gerais y São Paulo (Prata, inéd.)

Para la flora del Paraguay hasta el momento no se realizó aún una revisión del género, aunque fueron citadas 11 especies, 7 variedades y 1 forma (Clarke 1903; Barros, 1942, 1947; López, 1996; Kral \& Strong, 1999). Bulbostylis consanguinea y B. papillosa fueron mencionadas para Paraguay por Guaglianone (1996). Sin embargo para $B$. consanguinea no se mencionó ningún ejemplar que documente su presencia en Paraguay.

En cuanto a B. papillosa, sobre la base de la cita Barros (1945), este autor mencionó el ejemplar Hassler 10091, coleccionado en Paraguay como perteneciente a esta especie, pero al estudiarlo se pudo comprobar que se trata de B. junciformis (Kunth) C. B. Clarke.

Kral (1971, 1978) y Adams (1994) consideran a $B$. papillosa como sinónimo de $B$. junciformis, pero estas dos especies tienen diferencias suficientes como para mantenerlas independientes. En B. papillosa el aquenio es oblongo, las células de la superficie presentan paredes rectas, con una protuberancia central cónica, mientras que en $B$. junciformis el aquenio es obcónico, las células de la superficie del aquenio presentan paredes ondeadas, con una protuberancia central semiesférica (Fig. 7 C-F).

\section{Materiales y Métodos}

Para la observación de los frutos en Microcopio Electrónico de Barrido (MEB) se extrajeron frutos de ejemplares de herbario, se montaron en una platina de cobre y fueron metalizados con oro paladio. Se utilizó el microscopio JEOL 5800 LV de la Secretaría General de Ciencia y Técnica de la Universidad Nacional del Nordeste. La observación de los frutos se realizó a $15 \mathrm{kv}$ y se tomaron fotografías digitales.

\section{Resultados}

\section{Bulbostylis svensoniana Steyerm.}

Fig. 1 y 6 (A-B)

Steyermark, J. A., Fieldiana, Bot. 28: 37, Fig. 5. 1951. Typus: Venezuela. Bolivar, on dry flat savanna with Curatella, between Upata and Rio Caroni, 400 m, 2 Aug 1944, Steyermark 57629 (holotypus F).

Hierba perenne, cespitosa, de 0,50-1,20 m alt., de base engrosada, con pelos castañoclaros a rojizos. Tallo de 1-2 mm diám., cilíndrico, estriado, glabro. Hojas de 7-40 cm long. x 0,03 cm lat., 1/3-1/2 del largo del tallo, circinadas; vaina 3-13 cm long., membranácea, castaño-clara, ápice oblicuo, con cilias de 4-5 cm long., lámina filiforme, cara abaxial estriada, 3-nevada, cara adaxial cóncava, márgenes escabrosos. Brácteas involucrales 5-6, foliáceas, glabras, base ensanchada, bráctea inferior igual o superando la inflorescencia. Inflorescencia de 6-9 cm long. x 2-6 cm lat., antelodio compuesto, ejes erectos con un fascículo terminal de espiguillas. Espiguillas de 4-5 mm long. x 1-1,2 mm lat., ovoides, 4-6-floras, agudas, sésiles. Glumas persistentes, de 2-2,5 mm long. $x$ 1- 1,2 mm lat., lanceoladas, naviculares, membranáceas, castaño-claras, carena uninervia, ápice agudo, mucronado, superficie escabrosa, las 2 glumas basales estériles. Flores con 3 estambres, anteras ca. $1 \mathrm{~mm}$, apiculadas, estigmas 3. Aquenio de 0,8-0,5 mm long., trígono, obovoide, estilopodio persistente, umbonado, las células de la superficie son poligonales de paredes rectas, sin protuberancia central.

Fenología: Florece y fructifica de noviembre a mayo.

Ecología: Habita en campos cerrados con suelo rocoso.

Distribución geográfica: Venezuela, centro-oeste y sudeste de Brasil, este de Bolivia y Paraguay.

Material estudiado. BOLIVIA. Santa Cruz: 


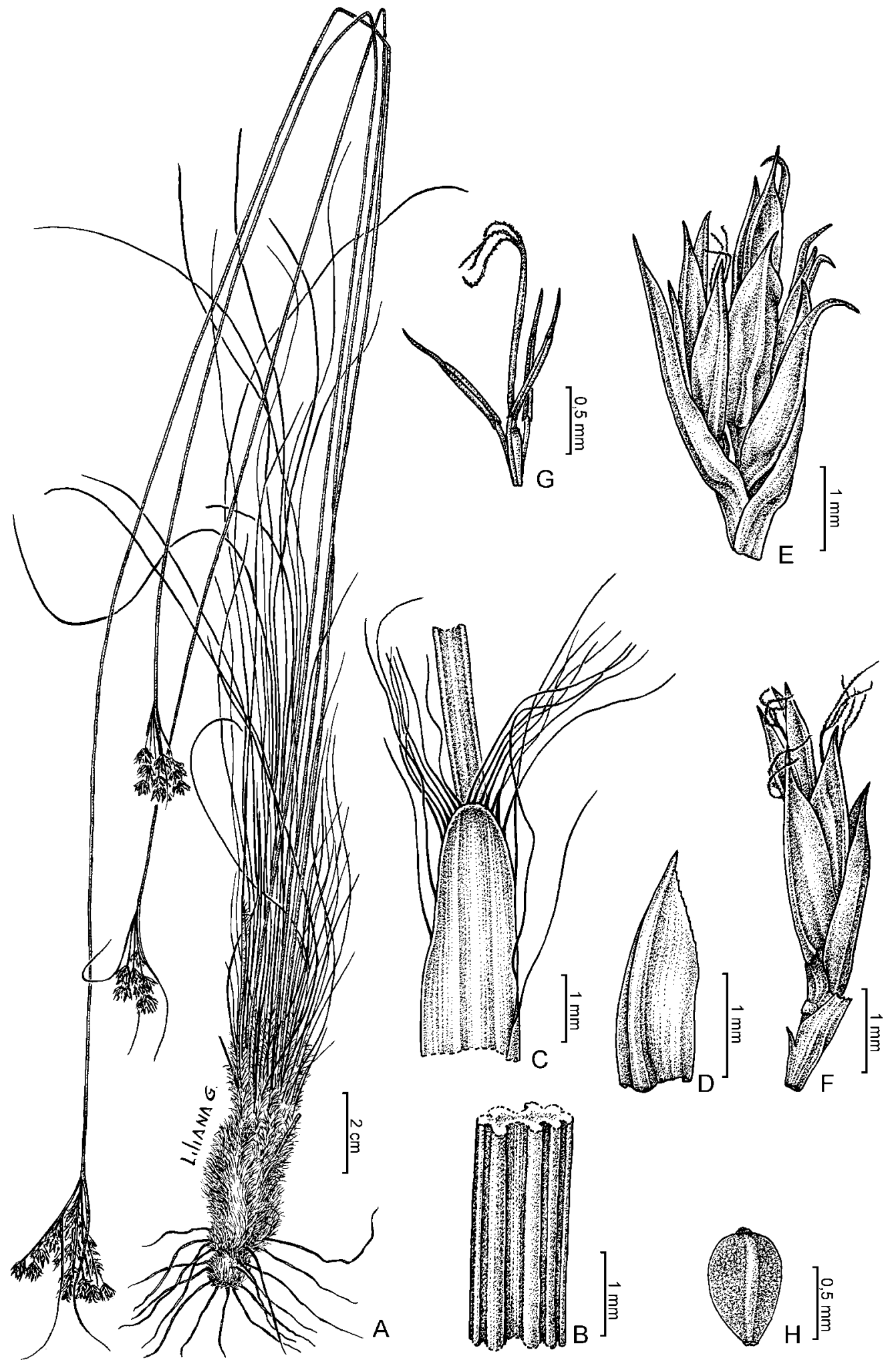

Fig. 1. Bulbostylis svensoniana. A: hábito. B: porción de tallo. C: ápice de la vaina. D: gluma, vista lateral. E: fascículo de espiguillas. F: espiguilla. G: flor. H: fruto (A-H, Solomon \& al. 6817). 
Prov. Chiquitos, camino Roboré-San José, 5 km NW de Roboré, 18²344”S, 59³9'19,5”W, Mostacedo \& al. 2845 (MO). Prov. Velasco, camino de San Rafael a San José, a 12,2 km S de San Rafael, 1652'57'S, 60³8'55”W, 355 msm, 1-II2005, Solis Neffa \& al. 1752 (CTES).

Obs.: Bulbostylis svensoniana es semejante a $B$. junciformis, de la que se diferencia por la base de la planta, que es engrosada, con pelos castaño-claros a rojizos, y por la forma y ornamentación de la superficie del fruto. En $B$. svensoniana el aquenio es obovoide, las células de la superficie son poligonales de paredes rectas, sin protuberancia central (Fig. 6 A-B), en B. junciformis el aquenio es obcónico-trígono y las células de la superficie son poligonales de paredes onduladas, con una protuberancia central (Fig. 7 E-F).

\section{Bulbostylis consanguinea (Kunth) C. B. Clarke}

Fig. 2 y 6 (C-D)

Clarke, C. B., Kew Bull. 8: 110. 1908.

Isolepis consanguinea Kunth, Enum. pl. 2: 211. 1837. Lectotypus, designado por Prata \& Thomas (inéd.): Brasilia. Sellow 2933 (holotypus K).

Oncostylis kunthiana Nees, in Mart., Fl. bras. 2(1): 87. 1842.

Hierba perenne, cespitosa, de 34-60(-100) cm alt., base castaña-clara o ferrugínea. Tallo de 30-57(-92) cm long. $x$ 0,8-1,5(-2) mm diám, verde-amarillento, con 12-19 costillas en transcorte, ligeramente antrorso-escábridas o glabras. Hojas de 8-22(-36) cm long., 1/ 2 a 1/3 del largo del tallo; vaina de $4-15 \mathrm{~cm}$ long., rojiza, lineada, antrorso-escabrosa, de ápice oblicuo, con cilias de 1,5-6 mm; lámina linear, de 0,5-18 cm long. x (0,5-)1-1,5 mm lat., cara adaxial levemente cóncava, cara abaxial 6-nervada, nervios y bordes antrorsoescábridos. Inflorescencia en antelodio compuesto, de (2-)3,5-9 cm long. x (1,5-)4,5-8 cm lat., espiguillas reunidas en 4-6(-9) fascículos, uno central sésil y los laterales en los extremos de ejes desarrollados, 1-3(-6) cm long., antrorso-escabrosos en toda la superfi- cie, cada fascículo formado por (3-)6-20 espiguillas. Involucro 4-6-filo, la bráctea inferior igual o ligeramente más larga que la inflorescencia, foliácea, de 3,5-6 cm long., ensanchada en la base, densamente antrorsoescabrosa, ferrugínea con 7 nervios y bordes hialinos. Espiguillas ovoideas, de 4,5-6 (-10) mm long. x 2-2,5 mm diám, 5-8(-15)-floras, bráctea inferior estéril, glumácea, mucronada. Glumas 2-3,7mm long. x 2-2,5 mm lat., ovadas, naviculares, obtusas, ferrugíneas con máculas pardas, superficie papilosa, bordes hialinos, irregularmente dentados con cilias en el ápice de los dientes, carena trinervia. Flores con estigma trífido, estambres 3, anteras de 0,5-1 mm long., agudas. Aquenio de 1-1,25 mm long. x 0,75 mm lat., obcónicotrígono, ápice truncado, atenuándose levemente en la base, superficie tuberculada, formando arrugas transversales, células rectangulares con bordes ondulados, castaño; estilopodio plano, castaño.

Fenología: Florece y fructifica desde septiembre a mayo.

Ecología: Habita en cerrado y suelo arenoso.

Distribución geográfica: Brasil, Paraguay (Amambay) y norte de Argentina (Formosa).

Material estudiado: PARAGUAY. Amambay. 25 km N de Pedro Juan Caballero, 18-IV-1995, Schinini \& al. 29252 A (CTES).

\section{Bulbostylis loefgrenii (Boeck.) Prata \& López}

Fig. 3 y 6 (E-F)

Prata, A. P. \& M. G. López, Kew Bull. 56: 1008. 2001.

Scirpus loefgrenii Boeck., Beitr. Cyper. 2: 16. 1890. Typus: Brasil. São Paulo, Feijão, Löfgren 976 (holotypus $\mathrm{B}$, isotypi $\mathrm{P}, \mathrm{SP}$ !, foto $\mathrm{K}$ ).

Hierba perenne, cespitosa, de $15-30 \mathrm{~cm}$ alt., base rojiza o castaña. Tallo filiforme, de 11,5-24 cm long. x 0,02-0,03 cm diám., con 5- 


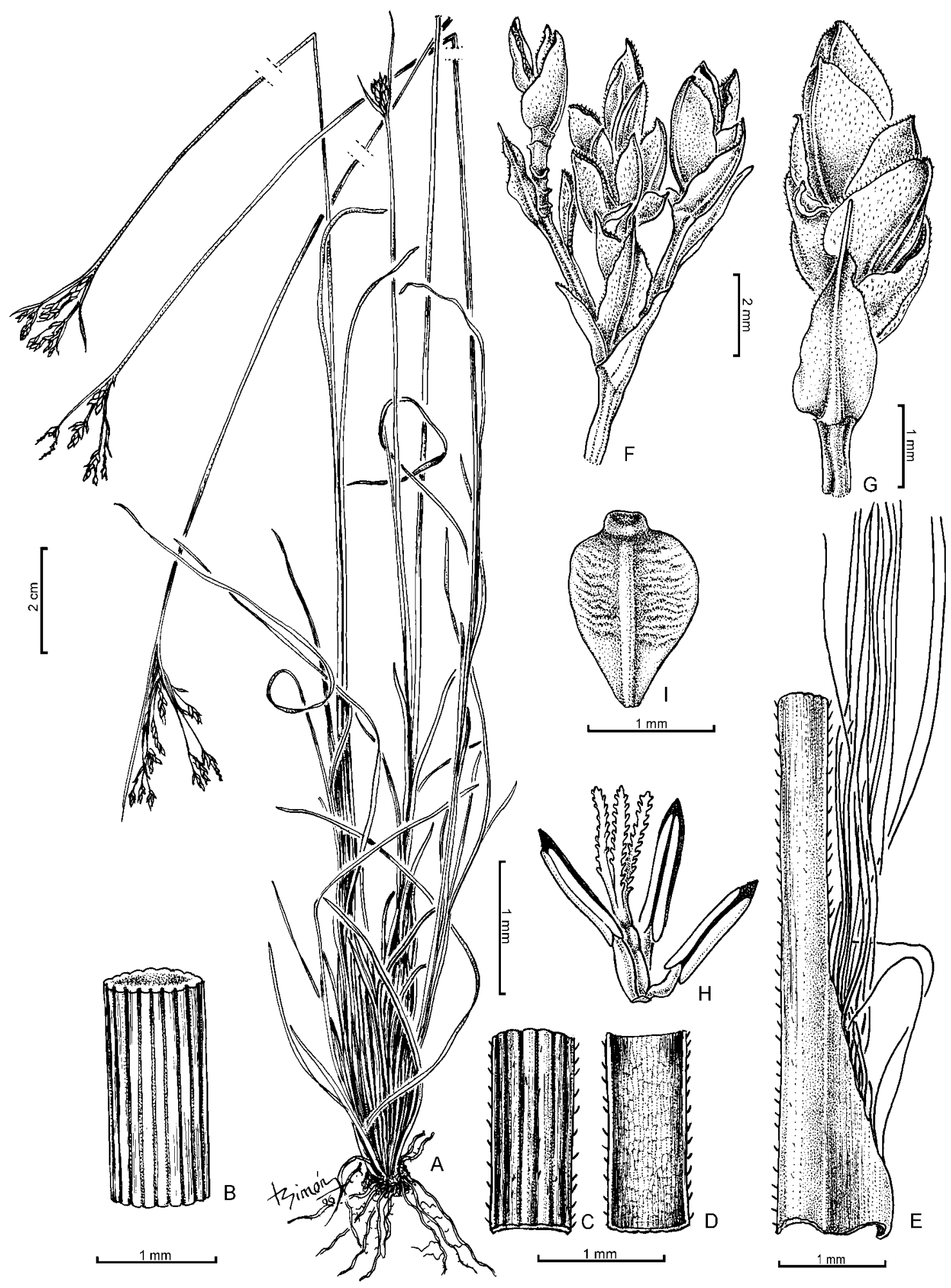

Fig. 2. Bulbostylis consanguinea. A: hábito. B: porción de tallo. C-D: porción de lámina, C: cara abaxial, D: cara adaxial. E: ápice de la vaina. F: fascículo de espiguillas. G: espiguilla. H: flor. I: aquenio (A-J, Schinini \& al. 29252 A). 


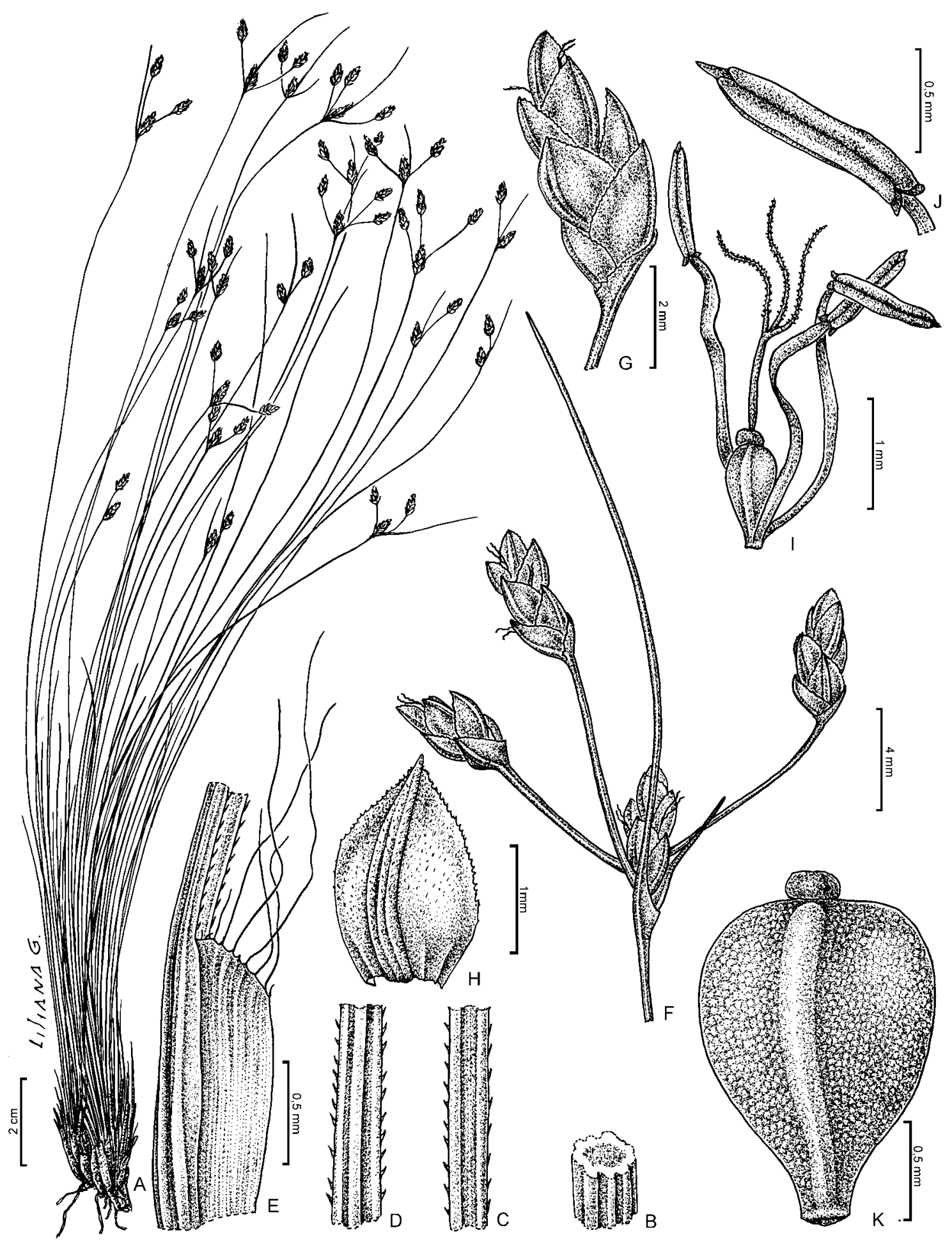

Fig. 3. Bulbostylis loefgrenii. A: hábito. B: porción de tallo. C-D: porción de lámina, C: cara adaxial, D: cara abaxial. E: ápice de la vaina. F: inflorescencia. G: espiguilla. H: gluma, vista dorsal. I: flor. J: antera. K: aquenio (A-K, Schinini \& al. 30181 A). 
8-costado, escábrido. Hojas de 6-10 cm long., 1/2 del largo del tallo; vaina de 1,7-3,2 cm long., castaño-claro, membranácea, ápice oblicuo, escasamente ciliada; lámina setácea, aguda, cara abaxial 3-nervada, cara adaxial cóncava, a veces plana, antrorso-escabrosa en los bordes. Inflorescencia en antelodio, generalmente simple a veces compuesto, de 0,7$2,5 \mathrm{~cm}$ long. $x$ 0,8-1,7 cm lat., con 2-4(-5) espiguillas, la central sésil. Involucro generalmente difilo, la bráctea más externa supera a la inflorescencia, de 1-3(-4) cm long., la siguiente de 0,2-0,7 cm long., filiformes, base ensanchada, glabras. Espiguillas de (0,8-)1,05,0 cm long., 5-8-floras, ovoides, agudas. Glumas ferrugíneas, de 1,9-2,5 x 1,8-2,4 mm, ovoides, naviculares, trinervadas, agudas, múticas, la inferior mucronada, membranáceas, margen hialino, ciliolado, escabrosa. Flores con estigma trífido, estambres 3, anteras de 0,8-1,3 mm long., lanceoladas, apiculadas. Aquenio de 1,5 x 0,8-1,2 mm, obovoide, superficie suavemente punteada formada por células pentagonales con una protuberancia en el centro, estilopodio castaño, claro, cilindroide.

Fenología: Florece y fructifica de agosto a marzo.

Ecología: Habita en cerrados, campo de suelo arenoso, suelo pedregoso y modificado.

Distribución geográfica: Sudeste de Brasil y Paraguay oriental.

Material estudiado: PARAGUAY. Concepción: N. Paraguay, zwischen Rio Apa und Rio Aquidaban, Centurion, XII-1908/1909, Fiebrig 5262 (G). San Pedro: S 23⒋'04,3" W 5317'34,8", 15-I-2002, Gonzáles 749 (CTES); 36 $\mathrm{km} \mathrm{N}$ de Tacuara, $24^{\circ} 22^{\prime} \mathrm{S}$, 56 $26^{\circ} \mathrm{W}$, Ea. La Manina, 21-X-1994, Krapovickas 45792 (CTES).

Caaguazú: $55^{\circ} 20^{\prime} \mathrm{W}, 24^{\circ} 35^{\prime}$ S, Cnia. Pindo, camino entre Itaquyry y Curuguati, estribaciones de la Sra. de San Joaquin, ca $300 \mathrm{msm}, 11-\mathrm{X}-1995$, Schinini 30181 A (CTES); 14 km N de Caaguazú, camino a Ihú, $56^{\circ} \mathrm{W}, 25^{\circ} 25$ 'S, $250 \mathrm{msm}$, 27-III1993, Schinini 28113 (CTES). Alto Paraná: 8 km $\mathrm{N}$ de Hernandarias, Tatí Yupí, 8 km E de la ruta, margen derecha del río Pirá Pitá. 7-II-2003, López
265 (CTES). Amambay: Ea. San Luis, Sierra de Amambay, cerca del límite Brasil-Paraguay,

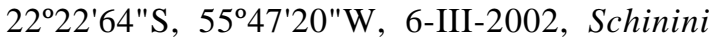
35805 (CTES); Parque Nacional Cerro Corá, camino al Cerro Trebol, XI-1992, Soria 5582 (CTES); Colonia Yvypyté, 56 ${ }^{\circ} \mathrm{W}, 23^{\circ} \mathrm{S}, 20-\mathrm{VIII}-$ 1980, Schinini20506 (CTES).

\section{Bulbostylis sellowiana (Kunth) Palla}

Fig. 4 y 7 A-B

Palla, E., Denkschr. Akad. Wien, Math.-Nat. 79: 179. 1908.

Isolepis sellowiana Kunth, Enum. pl. 2: 208. 1837. Typus: Brasil. São Paulo, An grasingen Stellen bei São Paulo, dem Meeresspiegel, VIII1901, Wettstein \& Schiffner (holotypus M, isotypi NY, C).

Scirpus sellowianus Boeck., Linnaea 36: 748. 1869/70.

Hierba perenne, cespitosa, de 21-78 cm alt., base fibrosa amarillenta, engrosada. Tallo de (13-)19-59 cm long. x 0,5-0,8 cm diám, 6-8(-10) estrías longitudinales marcadas, hispídulo, con pelos caducos cuando maduros. Hojas 6-30 cm long., 1/2-2/3 del largo del tallo; vaina de 0,9-3 cm long., ápice oblicuo, ciliado, cilias 2-8 mm long., membranácea; láminas setáceas, agudas, cara abaxial no estriada, cara adaxial plana, híspida. Inflorescencia capitada, de 0,5-1 x 0,8-1,3 cm, hemiesférica, 2-4 espiguillas. Involucro 2-4filo, brácteas ferrugíneas, la más externa $1 \mathrm{~cm}$ long., glumiformes, base ensanchada. Espiguillas ovoides, de 6-10 mm long. x 2,5-4 mm diám., ca.18-floras. Glumas castaño oscuro, de 3,8-4 mm long. x 1,3-2,1 mm lat., ovadas, ápice obtuso, naviculares, múticas, coriáceas, pubérulas. Flores con estilo trífido, de $4,5 \mathrm{~mm}$ long., estambres 3, anteras de 2,7-3 mm long., apiculadas con papilas apicales, agudas. Aquenio de 1,2-1,3 x 1,2 mm, obcordiforme, trígono, base atenuada, ángulos espesados, superfície con ondas horizontales levemente marcadas, estilopodio plano.

Fenología: Florece y fructifica durante todo el año. 


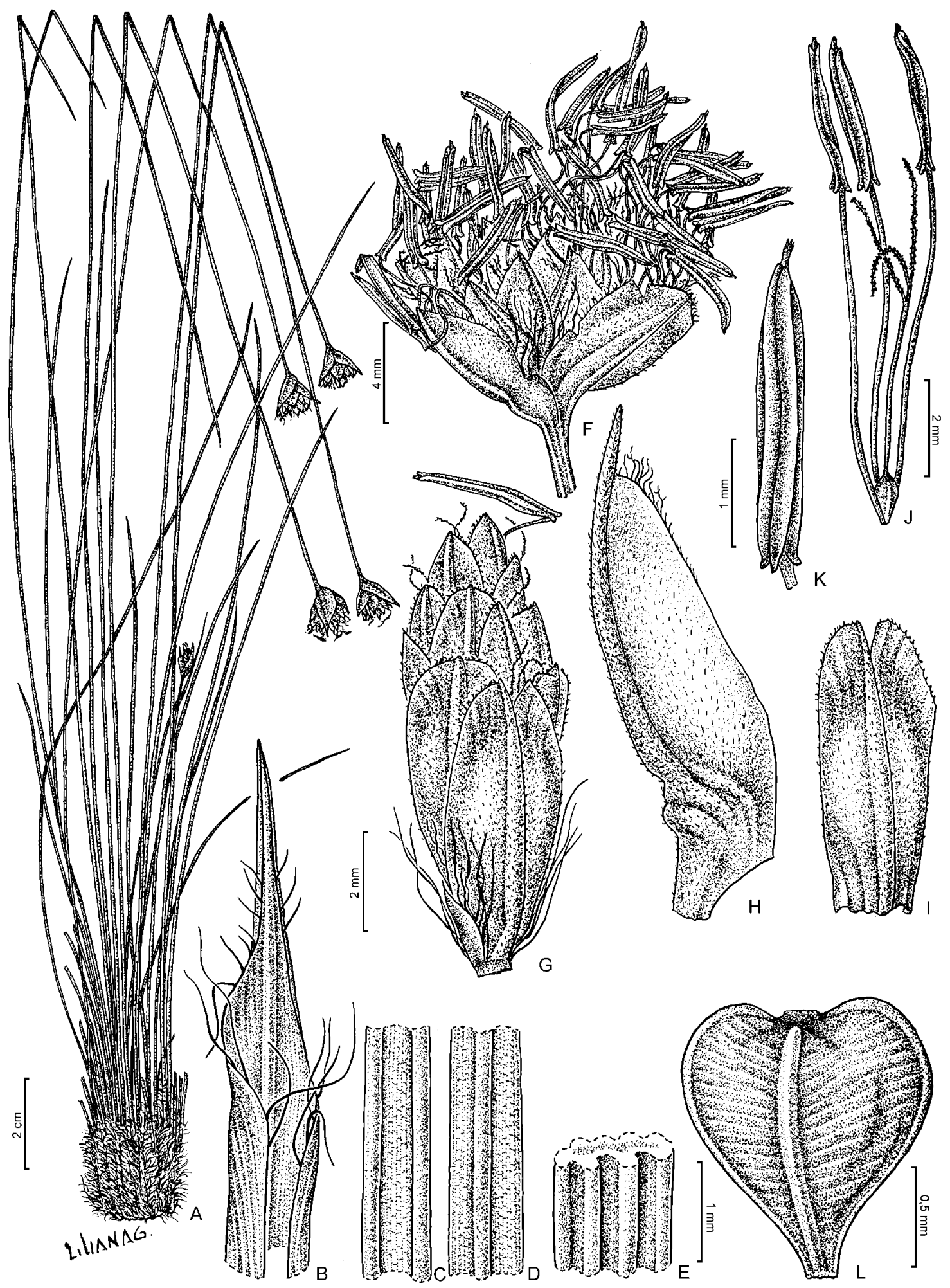

Fig. 4. Bulbostylis sellowiana. A: hábito. B: ápice de la vaina. C-D: porción de lámina, C: cara adaxial, D: cara abaxial. E: porción de tallo. F: inflorescencia. G: espiguilla. H: bráctea del involucro. I: gluma, vista dorsal. J: flor. K: antera. L: aquenio (A-L, Hassler 9429). 


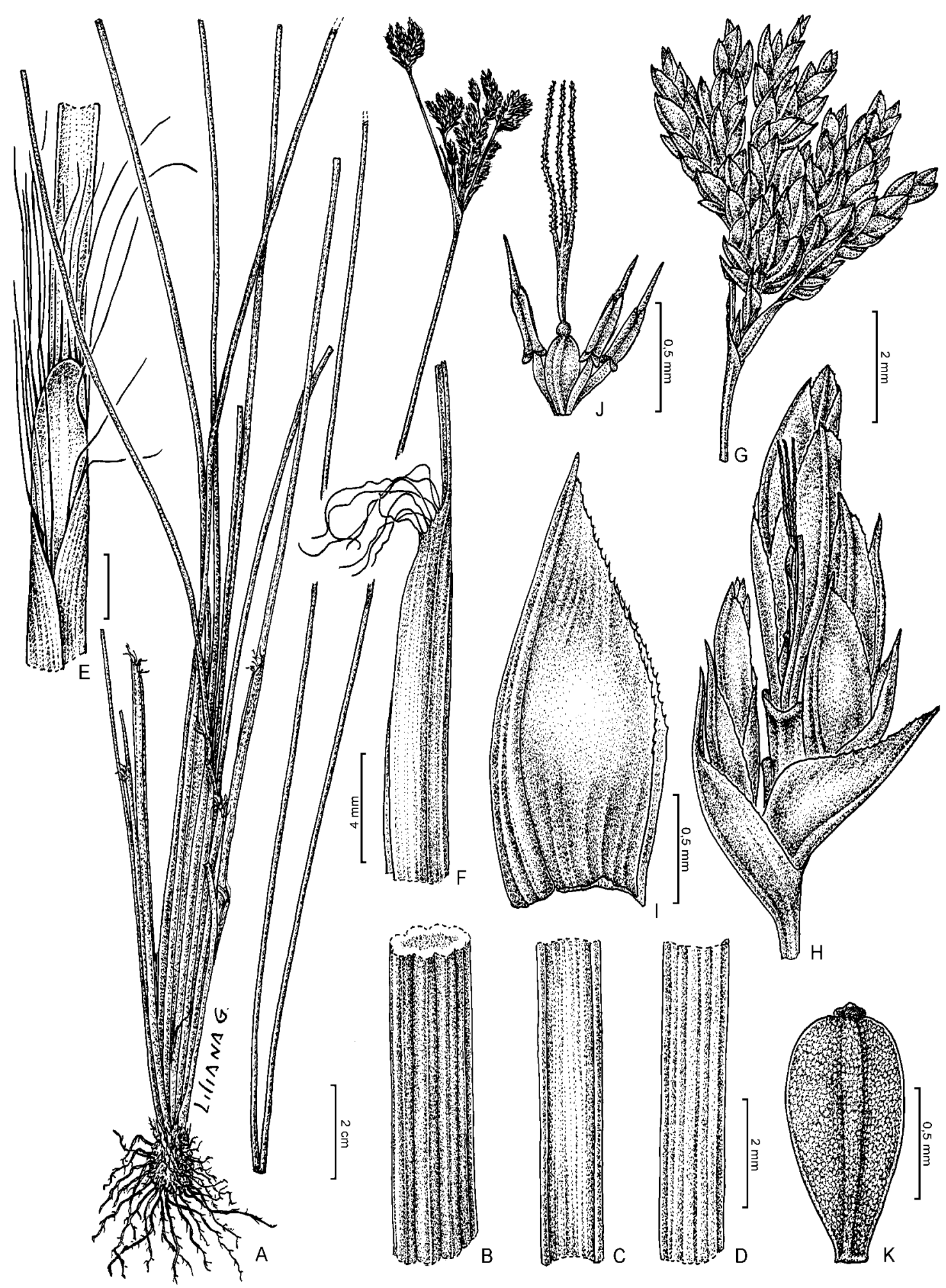

Fig. 5. Bulbostylis papillosa. A: hábito. B: porción de tallo. C-D: porción de lámina, C: cara adaxial, D: cara abaxial. E-F: ápice de la vaina, E: vista ventral, F: vista lateral. G: porción de inflorescencia. H: fascículo de espiguillas. I: gluma, vista lateral. J: flor. K: aquenio (A-K, Schinini \& al. 29318). 
Ecología: Habita en suelo inundable.

Distribución geográfica: Centro y sur de Brasil y Paraguay, dónde hasta el momento sólo fue encontrada en Caaguazú.

Material estudiado: PARAGUAY. Caaguazú: In regione fluminis Yhú, IX-1905, Hassler 9429 (G).

\section{Bulbostylis papillosa Kük.}

Fig. 5

Kükenthal, G., Repert. Spec. Nov. Regni Veg. 23: 198. 1926.
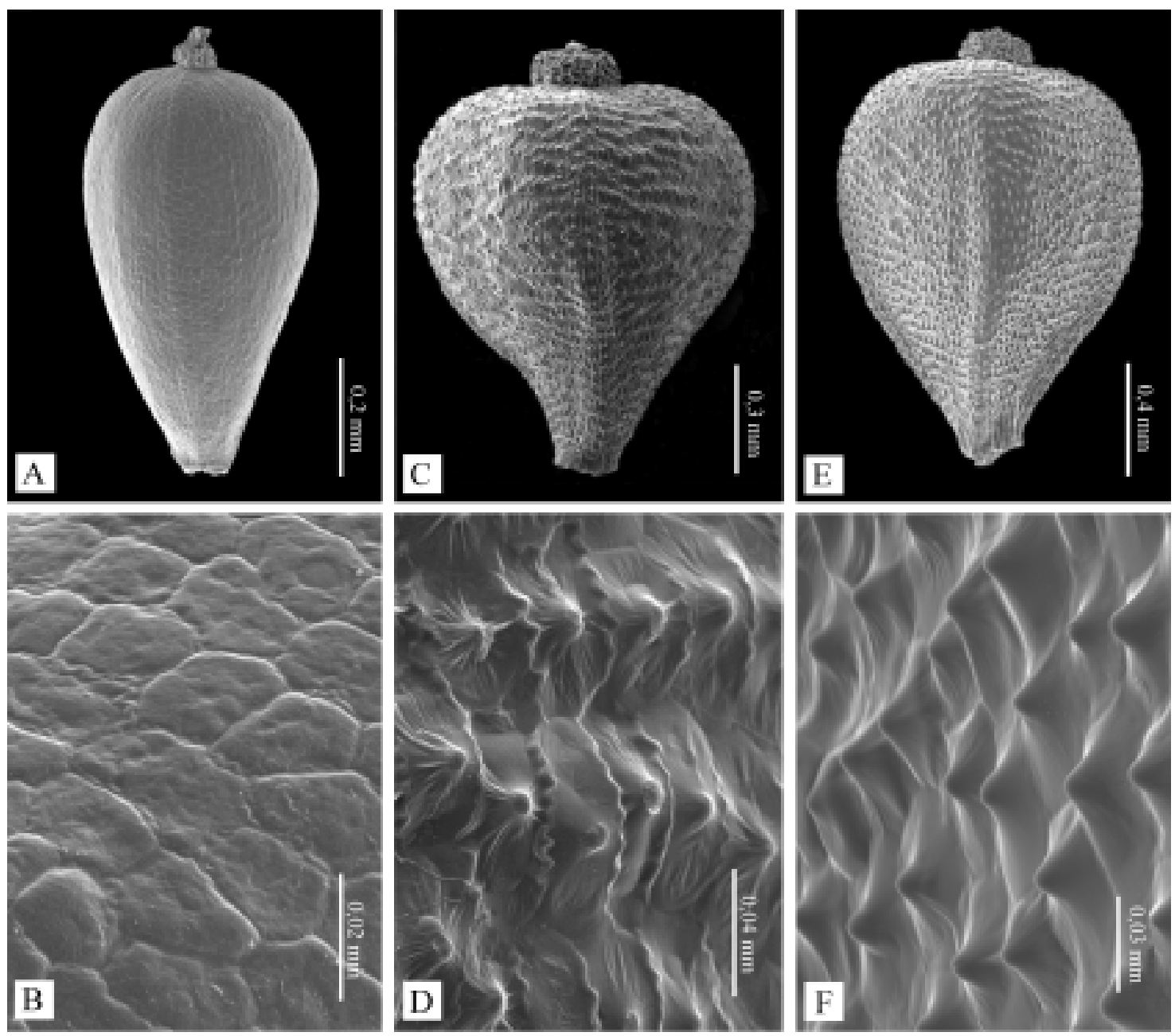

Fig. 6. Microfotografías con MEB de aquenios de Bulbostylis. A-B: B. svensoniana. A: fruto entero, B: detalle de la pared (A-B, Solomon \& al. 6817). C-D. B. consanguinea. C: fruto entero, D: detalle de la pared (C-D, Schinini \& al. 29252-A). E-F: B. loefgrenii. E: fruto entero, F: detalle de la pared (E-F, Fiebrig 5262). 

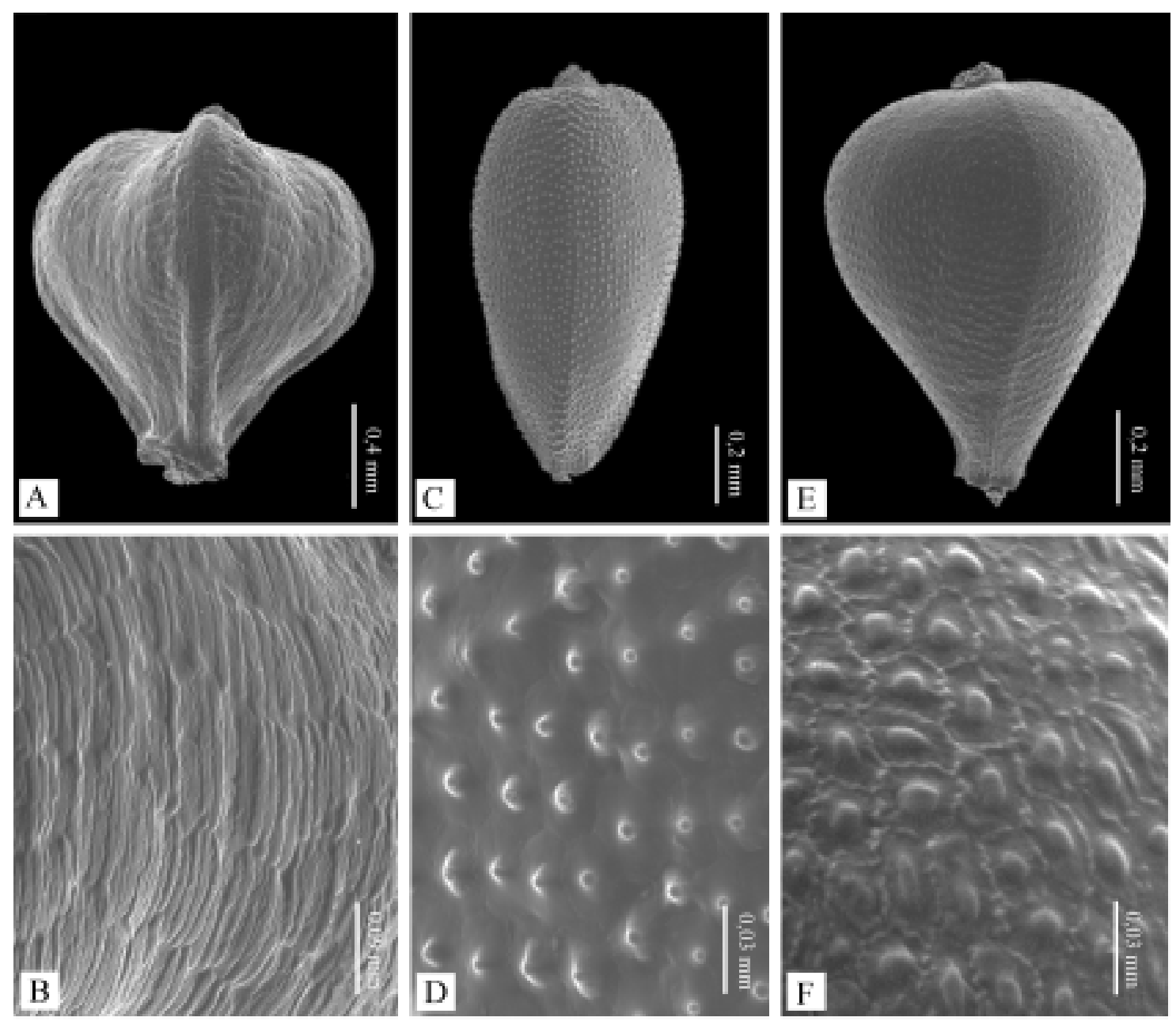

Fig. 7. Microfotografías con MEB de aquenios de Bulbostylis. A-B: B. sellowiana. A: fruto entero, B: detalle de la pared (A-B, Hassler 9429). C-D: B. papillosa, C: fruto entero, D: detalle de la pared (C-D, Schinini \& al. 29318). EF: B. junciformis. E: fruto entero, F: detalle de la pared (E-F, Pedersen 11023, CTES).

cencia en antelodio compuesto, denso, de 2-5 cm long. x 1,5-3,5 cm lat., espiguillas reunidas en fascículos, ejes de longitud variable, glabros, involucro más corto que la inflorescencia, brácteas foliáceas, base ensanchada, castaña con máculas ferrugíneas. Espiguillas ovoideas, de 2-6 x 1-1,5 mm, 8-10-floras, bráctea glumácea. Gluma de 1,5-2 mm long. $\mathrm{x}$ 1,4-1,6 mm lat., ovada, aguda, las inferiores mucronadas, ferrugíneas con máculas pardas, superficie escabrosa, carena trinervia. Flores con estigma trífido, estambres 3 , anteras de 2$4 \mathrm{~mm}$ long., conectivo de 1,5-3 mm long., con papilas agudas. Aquenio de 1,1-1,25 mm long. x 0,5-0,8 mm lat., oblongo-trígono, an- gosto, levemente atenuado hacia la base, ápice redondeado, superficie formada por células pentagonales de paredes rectas con una protuberancia central cónica, estilopodio umbonado, castaño-oscuro.

Fenología: Florece y fructifica en marzo y abril.

Ecología: Habita en campo cerrado y suelo inundable.

Distribución geográfica: México, Santo Domingo, Cuba, Brasil, Bolivia y Paraguay. 
Material estudiado: PARAGUAY. Amambay:

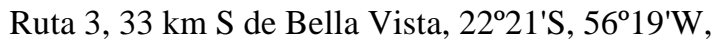
18-IV-1995, Schinini \& al. 29318 (CTES); 25 km N de Pedro Juan Caballero, 18-IV-1995, Schinini \& al. 29252 (CTES).

\section{Agradecimientos}

Quiero expresar mi agradecimiento a la Prof. Rosa Guaglianone, por su constante apoyo y la lectura del manuscrito. A Liliana Gómez y Laura Simón por la confección de las láminas.

\section{Bibliografía}

ADAMS, C. D. 1994. Cyperaceae. In G. Davidse, M. Sousa \& A. O. Chater (eds.). Fl. Mesoamer. 6: 452-455.

BARROS, M. 1942. Notas ciperológicas II. Darwiniana 6 (1): 122-124.

1945. Ciperáceas argentinas. IV. Géneros Fimbristylis, Bulbostylis, Fuirena, Dichromena, Schoenus, Oreobolus, Carpha, Rhynchospora, Scleria y Uncinia. Anales Mus. Argent. Ci. Nat.
“Bernardino Rivadavia 41: 323-479.

1947. Cyperaceae - Scirpoideae. In H. Descole (ed.). Gen. Sp. Pl. Argent. 4(2): 273-297.

CLARKE, C. B. 1903. Cyperaceae. In R. Chodat \& E. Hassler (eds.). Plantae Hasslerianae, enumeration des plantes récoltées au Paraguay par le Dr. E. Hassler de 1885 à 1902. Bull. Herb. Boissier 11, sér. 2: 1018-1019.

GOETGHEBEUR, P. 1998. Cyperaceae. In K. Kubitzki \& col. (eds.). The Families and Genera of Vascular Plants 4: 141-190.

GUAGLIANONE, E. R. 1996. Cyperaceae. In F. O. Zuloaga \& O. Morrone (eds.). Catálogo de las Plantas Vasculares de la República Argentina I. Monogr. Syst. Bot. Missouri Bot. Gard. 60: 130-133.

KRAL, R. 1971. A treatment of Abildgaardia, Bulbostylis and Fimbristylis (Cyperaceae) for North America. Sida 4 (2): 57-227.

1998. Bulbostylis (Cyperaceae). In J. A. Steyermark, P. E. Berry \& B. K. Holst (eds.). Flora of the Venezuelan Guayana 4: 505-514.

— \& M. T. STRONG. 1999. Eight Novelties in Abildgaardia and Bulbostylis (Cyperaceae) from South America. Sida 18 (3): 837-859.

LÓPEZ, M. G. 1996. Una nueva especie de Bulbostylis (Cyperaceae). Bonplandia 9 (1-2): 29-33.

PRATA, A. P. (inéd.). O gênero Bulbostylis Kunth (Cyperaceae) no Brasil. Tesis Instituto de Biociências da Universidade de São Paulo. 205 pp.

Original recibido el 31 de octubre de 2006; aceptado el 27 de noviembre de 2006. 